\title{
A Comparison of FDM Structural Layouts and Implementation of an Open-Source Arm-Based System
}

\author{
David Pollard ${ }^{1, a}$, Guido Herrmann ${ }^{2}$, Carwyn Ward ${ }^{3}$ and Julie Etches ${ }^{3}$ \\ ${ }^{1}$ Bristol Robotics Laboratory, University of the West of England, Coldharbour Lane, Bristol, BS16 1QY, UK \\ ${ }^{2}$ Department of Mechanical Engineering, University of Bristol, University Walk, Bristol, BS8 1TR, UK \\ ${ }^{3}$ Bristol Composites Institute (ACCIS), University of Bristol, University Walk, Bristol, BS8 1TR, UK
}

\begin{abstract}
Fused Deposition Modelling (FDM) is a manufacturing process to build components in a layer-by-layer approach through extrusion of polymers from a movable nozzle, allowing for significantly higher degrees of complexity over machined parts. Current FDM systems typically use actuation provided through a gantry or delta structural layout, operating through depositing successive planar layers in a $2.5 \mathrm{D}$ process; it has been shown in numerous studies the bonding between layers has significantly lower strength than the homogeneous material or in-plane properties - an issue which can be mitigated through the deposition of curved layers. This paper compares four differing structural layouts of FDM systems (gantry, delta, Stewart Platform, and arm-based) to identify the key advantages of an arm-based method as the increased workspace and manipulability enabling "Additive Finalisation" of components, and suitability for curved layer FDM. Details are then presented of the open-source implementation and evaluation of a 6 degree-of-freedom arm-based FDM printer at the University of Bristol.
\end{abstract}

\section{Introduction}

Additive Manufacture (AM) was defined in ISO/ASTM 52900 as a "process of joining materials to make parts from 3D model data, usually layer upon layer" [1]. Components produced through AM are constructed through fusion of raw materials, as opposed to subtractive manufacture where they are machined from a larger block. This provides numerous advantages over the traditionally machined parts, such as reduced material waste, increased design freedom, and suitability for economical lowvolume manufacture [2]. Fused Deposition Modelling (FDM) is a method of AM comprising of extrusion of a molten polymer through a movable nozzle, and was pioneered by Stratasys in 1992 [3]. Recent open-source developments and the cheap, non-toxic materials have enabled its use for desktop production of plastic components [2].

A majority of AM processes, including FDM, are based on a slicing procedure to generate a component; a CAD format file is provided, "sliced" into a stack of 2D layers, and a toolpath for the required material deposition is generated for each layer. The effect of layered manufacturing requires support structures for overhanging sections, and the "staircasing", where the layer thickness causes an uneven surface on sloping sections; this effect is visible in FDM where layer heights are typically greater than $0.1 \mathrm{~mm}$ [4]. Additionally, the part orientation greatly affects the final properties, as

\footnotetext{
${ }^{a}$ Corresponding author: d.pollard@bristol.ac.uk
} 
numerous studies have found the bonding between surfaces is significantly weaker than the material properties in the plane of the layer $[2,4]$.

Previous research efforts have investigated 3D layered manufacturing techniques for FDM. Singamneni et al. [5] evaluated curved layer deposition, with an investigation into toolpath generation for curved layers, and demonstrated improved surface quality and mechanical properties of a curved beam; the compressive load of the curved part under 3-point bending increased by $40 \%$ over the traditional 2.5D layered design. Multi-Directional Layered Deposition (MDLD) was explored by Singh et al. [6], where overhangs, which typically require support structures, were eliminated through changing the angle of the slicing plane relative to the build surface. This process allows for the division of a CAD model into supported and unsupported sections and, through varying the slicing orientation between sections, eliminated the requirement for printing of supports for overhanging sections. Such works demonstrate the advantages of printing in multiple dimensions, but limitations imposed by traditional gantry-style mechanisms limit the use of these systems.

This paper details an approach to adaptation of an industrial robotic arm for use in FDM research, to explore aspects of in-situ manufacture. A brief overview of academic multi-dimensional AM is first presented, followed by a comparison of structural layouts of existing FDM machines to identify the use cases for an arm-based FDM system. Section 4 provides an overview of the current implementation at the University of Bristol, using an ABB IRB 140 robotic arm, followed by examples of its performance. Finally, future developments are presented, along with details on access to the open source code developed during this project.

\section{Background}

To date, there have been a number of systems developed for multi-dimensional FDM based on parallel or serial manipulators. Song et al. implemented a parallel kinematic robot, with the extruder affixed to a 6 degree of freedom (DOF) Stewart Platform [7]; a platform comprising of 6 linear actuators affixed to the extruder. They demonstrated the capability to laser scan a surface, generate a toolpath to maintain an angle normal to the surface, and deposit material on a curved surface. A similar concept was trialled by Dröder et al. [8] demonstrating "Additive Finalisation", a concept where a massproduced base component has final geometric features added using FDM - with use of a Stewart Platform, they were able to add features onto existing surfaces. A similar project equipped an opensource 3D printer with two additional degrees of freedom, and a laser scanner [9], allowing the identification of a surface, and superimposition of a CAD object to generate a conforming toolpath for deposition onto the scanned surface, and final build - it was noted there is a significantly higher complexity for slicing curved layers over the traditional planar layers due to the possibility of collision with the previously deposited structure.

MIT implanted an early instance of robotic arm-based AM, through use of a Kuka arm with changeable end effectors allowing for foam extrusion, a milling tool to machine the foam to a fine finish, and the FDM of polymers [10]. This robot was operated through generation of the toolpath through a traditional slicing program, and conversion into the Kuka Robot Language file for operation. A second implementation of FDM on a robotic arm is discussed in [11], where a Motoman robot arm demonstrated multi-plane deposition. The commands were produced in the format of GCode, with additional rotational parameters for nozzle orientation, and communicated from the PC to the robot via the MotoCom SDK libraries.

\section{Comparison of 3D printing system structures}

To identify the merits of robotic arm-based printing, an accuracy comparison was performed between three structural layouts, as presented in Table 1. The Ultimaker 3 [12] and Rostock Max V3 [13] were used as representative machines of the gantry and delta classes respectively, and the 6 DOF ABB IRB 140 [14], as used in Section 4, to compare the arm-based printer. As the Stewart Platform-based 
printers were only discussed in the literature, data was not available and so discussed below to aid comparison.

Table 1: Comparison of structural layouts suitable for FDM

\begin{tabular}{|l|l|l|l|}
\hline & Gantry [12] & Delta [13] & Arm [14] \\
\hline Positioning Accuracy $(\mu \mathrm{m})$ & 125 & (variable) & 30 \\
\hline Max speed $(\mathrm{XY})\left(\mathrm{mm} \mathrm{s}^{-1}\right)$ & 300 & 300 & $1000+$ \\
\hline Max speed $(\mathrm{Z})\left(\mathrm{mm} \mathrm{s}^{-1}\right)$ & 5 & 300 & $1000+$ \\
\hline Build area / printer footprint & 0.356 & 0.336 & 22.2 \\
\hline
\end{tabular}

It was noted there are no standardised methods for assessing the accuracy of FDM machines. A common method of stating accuracy is based upon the stepper motor resolution and associated gearing, with the gantry layout achieving $12.5 \mu \mathrm{m}$ accuracy in the $X Y$, and $2.5 \mu \mathrm{m}$ in $Z$, as described by the manufacturers [12]. However, upon examination of a similar printer, Weiss et al. [15] identified a mean error during a trajectory to be $400 \mu \mathrm{m}$ at a speed of $90 \mathrm{~mm} \mathrm{~s}^{-1}$. No data could be found for the accuracy of the delta printer, but uses gearing similar to the XY stage of the gantry printer. The Stewart Platform system used by Song et al. [7] had a stated accuracy of $<500 \mu \mathrm{m}$, and Dröder et al. [8] note the accuracy of their implemented Stewart Platform printer remained slightly lower than a comparable 3D printer; this may be partially due to the difficulties in implementing inverse kinematics for the high DOF parallel system. In contrast, the robot arm has significantly better positioning capabilities, due to its development for industrial applications. The stated accuracy when following a path is significantly lower, at $670 \mu \mathrm{m}$, as discussed in Section5.

Table 1 shows the key difference between the gantry and delta printers is the capability of fast movement in the $Z$ axis - as the former has a single stepper motor for each axis, the $X Y$ positioning is controlled by a belt drive, and the $Z$ through a lead screw, increasing the accuracy but decreasing the maximum speed. At typical print speeds, the slower $Z$ axis speed of the gantry inhibits the printing of curved layers; it was for this reason Allen et al. to use a delta printer during their investigations into curved layer deposition [16]. Similar to a delta printer, the robot arm is capable of rapid movements throughout its workspace, as an FDM nozzle would typically be within the payload range of the arm.

Finally, the ratio of build area to the printer footprint is relatively low for both the Gantry and Delta robot. The calculation in Table 1 was for the robot arm was performed with the $X Y$ plane intersecting the base of the robot, as the reachable workspace varies with $Z$, as shown in Figure 1 . While the Delta robot is capable of rapid movement in the $Z$ direction required for printing curved layers, the inability to position the nozzle normal to the substrate constrains the maximum printed gradient to be dependent on the nozzle geometry. The Stewart Platforms proposed in [7, 8] allow for repositioning, but exhibit a strong positional and orientation coupling.

Through the comparison of the four structural layouts for FDM, use cases for arm-based FDM can be identified as increased manufacturing speed of larger components through utilisation of higher achievable speeds over the workspace - this would require larger diameter nozzles, reducing achievable quality but increasing deposition rates. "Additive Finalisation" would be a second use case, presented by Dröder et al. [8], where the base component could be placed on a conveyor belt adjacent to the robot arm, allowing the arm to locate and print on the surface. A major consideration for its industrial use would be the value added, as a robotic arm would represent a significantly higher investment than a commercial printer, and as such its corresponding capabilities must be higher. 


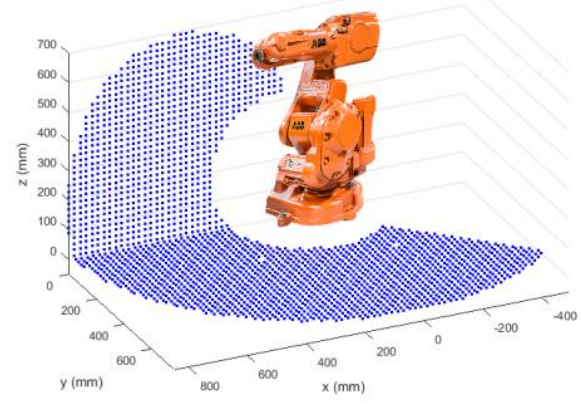

Figure 1: Workspace of IRB 140 robot arm constrained to keeping the end effector normal to the $X Y$ plane, as used for conventional FDM. Removing the constraint for angle orientation would allow an increased workspace.

\section{System design}

The robotic system at the University of Bristol was implemented on a 6 DOF ABB IRB 140 robotic arm, which has a $6 \mathrm{~kg}$ payload capability, $0.8 \mathrm{~m}$ reach, and a pose repeatability of $0.03 \mathrm{~mm}$. The robot cell also contained a 2 DOF IRBP A250 workpiece positioner, and was controlled through an ABB IRC5 controller running RobotWare 5.15. The relevant options enabled on the robot are the 616-1 PC Interface, and a DSQC 652 digital I/O module. An Arduino, connected to a RAMPS 1.4 breakout board, was used to control the extruder temperature and a stepper motor for filament feed. This was connected to the DSQC I/O module via a voltage divider, reducing the voltage from the $24 \mathrm{~V}$ signal to $4.8 \mathrm{~V}$ suitable for the Arduino. The extruder is comprised of a BondTech dual drive extruder, directly mounted to an E3D V6 hotend with a $0.6 \mathrm{~mm}$ nozzle - selected to enable higher speed printing. Figure 2 shows the system architecture, detailing the key operational functions.

OpenABB [17] was a software package developed for controlling ABB robots through Python, $\mathrm{C}++$, or ROS (Robotic Operating System) released with the MIT License. The implemented code was composed of two parts; a server module written in RAPID, the native language implemented within the IRC5 controller, and a Python class implemented on the controlling PC. To counter the lag of the Ethernet socket communication between the PC and IRC5, movements within a toolpath were sent in a buffer, providing the IRC5 with the coordinates for the execution of a full path to avoid pauses whilst awaiting further position data. As similar paths are often repeated for successive layers in FDM, functions were implemented to save, load, and offset the buffer to reduce time spent communicating coordinates.

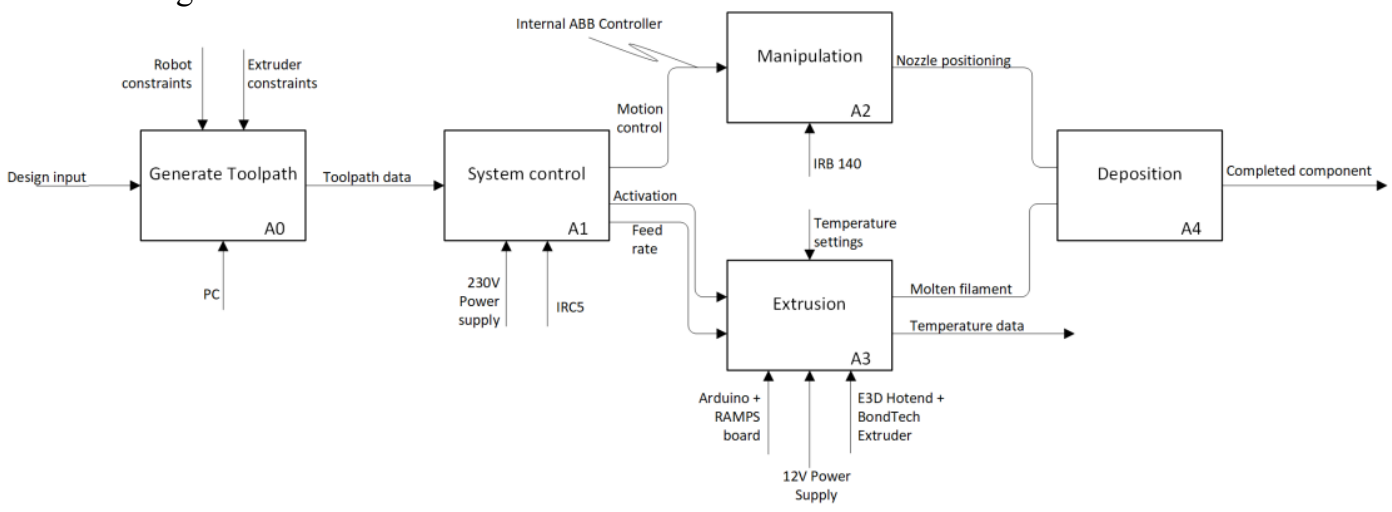

Figure 2: Diagram based on IDEF0 detailing key operations of the robotic system. 
The extruder activation signal was supplied by the IRC5 robot controller to ensure synchronisation with the robot motion. To adjust the feed rate for the current path, a signal was passed to the extruder controller through a Group Output; a collection of eight digital signals to representing an 8-bit unsigned integer in the range $[0,255]$. The Arduino controlling the extruder was connected to the operator's PC to allow monitoring of extrusion temperature, and adjustment of extrusion settings; this allows the testing of difference control systems, such as those examined by these authors in [18].

\section{Results and Discussion}

As previously discussed, the stated accuracy of the ABB IRB 140 arm for linear path following is 670 $\mu \mathrm{m}$, with a pose accuracy of $30 \mu \mathrm{m}$ [14]. This reduces the theoretical accuracy, but it should be noted that the linear path following accuracy was tested under ISO 9283, where the error was measured and averaged over a range of tool speeds - previous research has shown a significant increase in trajectory following error with speed [19]. Upon implementation, there was no noticeable error along layer lines due to the low theoretical accuracy in the $Z$ direction - potentially due to the relatively low speeds used during the printing process, as opposed to the maximum speeds used during the manufacturers tests, allowing for improved trajectory following. The limiting factor on maximum print speed was the inter-layer adhesion, with deposited material peeling from the substrate.

Figure 3 shows two objects produced through the robotic arm FDM process; a rectangular section (left), and a repair patch to a composite panel demonstrating the ability to print within a confined space on an angled surface. During printing, the average time to send a within a buffer was $0.08 \mathrm{~s}-$ this could be reduced through consistent orientation throughout a movement to reduce the data sent. A key issue found was the accuracy during the start and stop of the extrusion process, due to the overextrusion during the IRC5 processing time. These errors were minimised with the addition of a vertical movement at the end of an extrusion process.
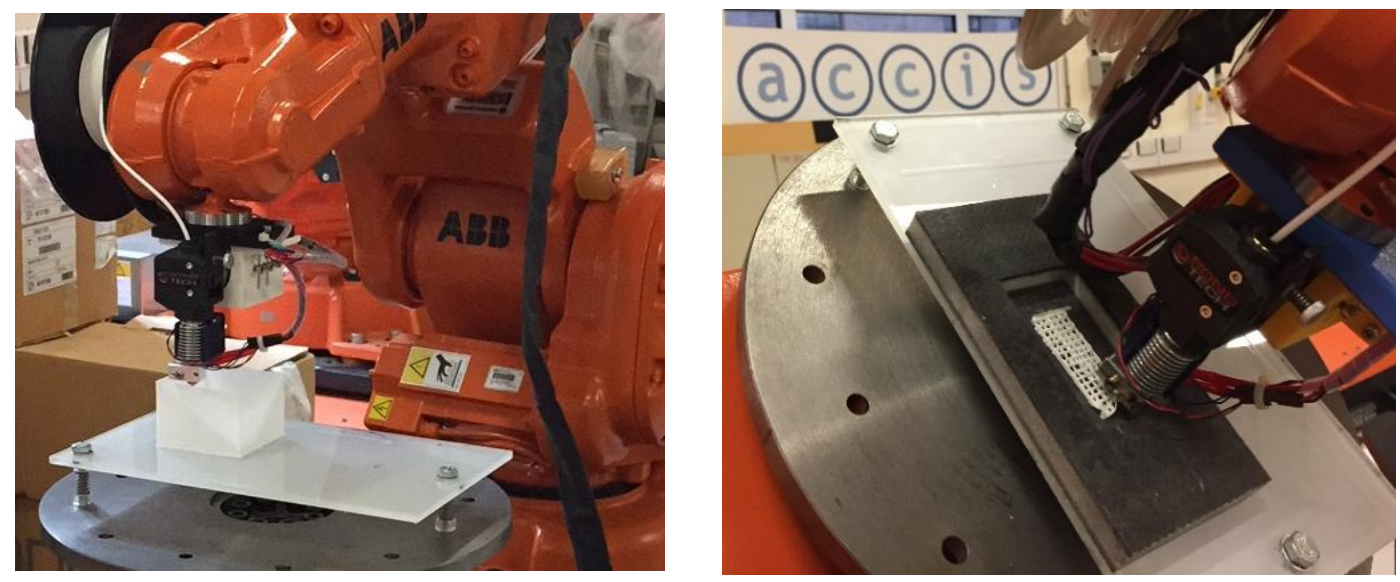

Figure 3: Examples of components produced through FDM with the robotic arm. Left shows the printing of a large component, and right demonstrates the printing of a repair patch with a composite sandwich panel as a $45^{\circ}$ angle.

\section{Conclusions and Further Work}

This work has examined the different structural layouts used for FDM (gantry, delta, Stewart Platform, and arm), finding the gantry platform has more consistent printing accuracy over the build 
area, and the delta platform has improved $Z$ axis speed, at the cost of an increased volume. The Stewart Platform allows for multi-DOF printing, but at the cost of a reduced workspace, and the arm is capable of rapid movements, higher manipulability, and a workspace significantly larger than its footprint, a factor not exhibited by other structural layouts for FDM. Based on this criteria, it was concluded arm-based FDM printers are suited for higher speed manufacturing of larger components, and "Additive Finalisation".

An implementation of FDM on a 6 DOF robotic arm, in this case an ABB IRB 140, was then presented, describing the hardware setup and a summary of the software communication protocol. Examples of the robot operation were presented, and its flexibility to print on slanted surfaces shown. The control software, based on the OpenABB architecture, used to operate this robot was released under the MIT license, and is available at:

https://github.com/bristolroboticslab/OpenABB-FDM

A number of studies are currently underway utilising the developed platform, such as the adaptation of toolpaths to orient the nozzle for printing on curved surfaces whilst avoiding edge collisions, and work on additive finalisation. Implementation improvements will investigate improvements in extruder control through current tool speed feedback. Additionally, the trade-off between system cost and capability will be explored to identify minimum performance requirements.

\section{Acknowledgements}

This work was supported by the EPSRC Centre for Doctoral Training in Future Autonomous Robotic Systems (FARSCOPE) at the Bristol Robotics Laboratory (grant EP/L015293/1).

The date necessary to support the conclusions are included within this paper, and the control software developed over the course of this project is available at https://github.com/bristolroboticslab/OpenABB-FDM.

\section{References}

1. ISO/ASTM 52900:2015, (2015)

2. W. Gao et al., Comput Aided Des, 69, 65-89, (2015)

3. S. S. Crump, US5121329A, (1992)

4. W. Oropallo, L. A. Piegl, Eng Comput, 32, 1-14, (2016)

5. S. Singamneni, A. Roychoudhury, O. Diegel, B. Huang, J Mater Process Tech, 212, 27-35, (2012)

6. P. Singh, Y. Moon, D. Dutta, S. Kota, Solid Freeform Fabr Symp Proc (2003)

7. X. Song, Y. Pan, Y. Chen, J. Manuf. Sci. Eng., 137, 021005-021005-13, 2015.

8. K. Dröder, J. K. Heyn, R. Gerbers, B. Wonnenberg, F. Dietrich, Procedia CIRP, 55, 122-127, (2016)

9. N. Bausch, D. P. Dawkins, R. Frei, S. Klein, IFAC-PapersOnLine, 49, 583-590, (2016)

10. S. Keating, N. Oxman, Robot Comput Integr Manuf, 29, 439-448, (2013)

11. I. B. Ishak, J. Fisher, P. Larochelle, IDETC/CIE 2016, (2016)

12. Ultimaker, "Ultimaker 3 specifications," [Online]. Available: https://ultimaker.com/en/products/ultimaker-3/specifications. [Accessed 1612 2017].

13. SeeMeCNC, "Rostock Max V3," [Online]. Available: https://www.seemecnc.com/products/rostockmax-v3-desktop-3d-printer-rtp. [Accessed 1612 2017].

14. ABB AB, Robotics, "Product specification IRB 140," (2017)

15. B. Weiss, D. W. Storti, M. A. Ganter, Rapid Prototyping J, 21, 482-490, (2015)

16. R. J. A. Allen, R. S. Trask, Additive Manuf., 8, 78-87, (2015)

17. M. Dawson-Haggerty, “OpenABB,”[Online]. Available: https://github.com/robotics/open_abb. [Accessed 2811 2017].

18. D. Pollard, C. Ward, G. Herrmann, J. Etches, Procedia Manuf., 11, 536-544, (2017)

19. M. Slamani, A. Nubiola, I. Bonev, Ind Rob, 39, 57-68, (2012) 